\title{
Tumor-derived exosomes: immune properties and clinical application in lung cancer
}

\author{
Jing Wu, Suyao Li, Pengfei Zhang \\ Department of Medical Oncology, Zhongshan Hospital, Fudan University, Shanghai 200032, China.
}

Correspondence to: Dr. Pengfei Zhang, Department of Medical Oncology, Zhongshan Hospital, Fudan University, 180 Fenglin Road, Shanghai 200032, China. E-mail: pfz@fudan.edu.cn

How to cite this article: Wu J, Li S, Zhang P. Tumor-derived exosomes: immune properties and clinical application in lung cancer. Cancer Drug Resist 2022;5:102-13. https://dx.doi.org/10.20517/cdr.2021.99

Received: 19 Sep 2021 First Decision: 22 Nov 2021 Revised: 6 Dec 2021 Accepted: 22 Dec 2021 Published: 8 Feb 2022

Academic Editors: Godefridus J. Peters, Chunxia Su Copy Editor: Yue-Yue Zhang Production Editor: Yue-Yue Zhang

\begin{abstract}
Lung cancer is the leading cause of cancer-related death worldwide. Despite advances in diagnosis and treatment of lung cancer, the overall survival remains poor. Evidence indicates that lung cancer development is a complex and dynamic process that involves interactions between tumor cells and their microenvironments, including immune cells. Exosomes are small extracellular vesicles secreted by most cell types; they contain functional molecules that allow intercellular communication. Tumor-derived exosomes (TEXs) carry both immunosuppressive and immunostimulatory mediators and may be involved in various immunomodulatory effects. TEXs, which partially mimic profiles of the parent cells, are a potential source of cancer biomarkers for prognosis, diagnosis, and prediction of response to therapy. In addition, TEXs may interfere with immunotherapies, but they also could be used as adjuvants and antigenic components in vaccines against lung cancer. In the context of lung cancer, identifying TEXs and understanding their contribution to tumorigenesis and the response to immunotherapies represents a challenging research area.
\end{abstract}

Keywords: Lung cancer, tumor-derived exosomes, cancer biomarkers, immunotherapies

\section{INTRODUCTION}

Lung cancer is one of the most common malignant tumors with the highest morbidity and mortality worldwide $^{[1]}$. Recently, immunotherapies have shown more effectiveness than traditional chemotherapy, 
and they have dramatically changed the treatment paradigm for lung cancer ${ }^{[2-4]}$. However, only a small proportion of patients can benefit from the immunotherapies, with primary and secondary resistance complicating treatment. One potential explanation for this phenomenon is the complexity and diversity of the tumor microenvironment (TME). Interactions of lung cancer cells with the surrounding TME are critical to cancer progression and response to immunotherapy. In recent years, the communication mediated by exosomes has extensively gained attention.

Exosomes are small bilayer membrane vesicles with a size of 30-100 $\mathrm{nm}$ in diameter that are secreted by various cell types such as tumor cells, immune cells, and fibroblasts ${ }^{[5,6]}$. Exosomes derived from tumor cells are referred to as tumor-derived exosomes (TEXs) ${ }^{[7]}$. TEXs have been shown to contain a variety of biomolecules including nucleic acids, proteins, enzymes, and lipids, which are involved in cancer progression, intercellular communication, and immunological function ${ }^{[8]}$. Studies have increasingly indicated that the number and composition of TEXs can in part reflect their cells of origin and biological state, which may serve as potential biomarkers in diagnosis and prognosis of cancer ${ }^{[9-11]}$. TEXs may affect cancer immunotherapy either by sequestration of therapeutic antibodies or supplying self-antigen carriers to improve cancer vaccine efficacy. Their biological roles in cancer progression as well as cancer immunotherapy and biomarkers have indicated that TEXs are critical components of the TME.

In this review, we first describe how TEXs are formed and released to the extracellular matrix, and discuss the composition of TEXs. Then, we outline the immunomodulatory function of TEXs in the lung cancer microenvironment. Moreover, we focus on the utility of TEXs as diagnostic and prognostic biomarkers in lung cancer. Finally, the recent findings on TEXs in immunological changes during immunotherapy are discussed.

\section{THE FORMATION, RELEASE, AND COMPOSITION OF TEXS}

TEXs, released by tumor cells, are present ubiquitously in tumor tissues and body fluids ${ }^{[12]}$. Exosome biogenesis initiates from the production of early endosomes via the internalization of membrane microdomains. The limiting membrane of early endosomes bud inwardly to form intraluminal vesicles, then becoming the multivesicular bodies. Finally, exosomes are released when multivesicular bodies fuse with the plasma membrane ${ }^{[13]}$. This formation of exosomes is a tightly regulated process; it involves two pathways through an Endosomal Sorting Complex Required for Transport (ESCRT)-dependent machinery or an ESCRT-independent machinery ${ }^{[14]}$. Once exosomes released, they are able to transfer information to their recipient cells through three main ways: endocytosis/phagocytosis, direct fusion with cellular membrane, and receptor-ligand interactions ${ }^{[13]}[$ Figure $1 \mathrm{~A}]$.

TEXs consist of a lipid-protein bilayer membrane, including membrane transport and fusion proteins (e.g., annexins, Rab proteins, and flotillin), MHC (class I and II molecules), adhesion molecules (e.g., ICAM, EPCAM, CD44, and integrins), inhibitory ligands [e.g., FasL, TRAIL, PD-L1, and transforming growth factor (TGF)- $\beta / \mathrm{LAP}$, tetraspanins (e.g., CD9, CD63, CD81, and CD82), tumor associated antigens, chaperones [e.g., heat-shock protein (HSP) 70 and HSP90], lipids, and glycolipids ${ }^{[12,13,15-17]}$. In their lumen, TEXs carry a variety of multivesicular bodies biogenesis proteins [e.g., Alix and tumor susceptibility gene 101 (TSG101)], cytoskeletal proteins (e.g., actin, tubulin, and vimentin), histones, oncoproteins, soluble factors, enzymes, cytokines, signaling molecules, and nucleic acids (including DNA, mRNA, and miRNA $)^{[13,15]}$ [Figure 1B]. The TEXs molecular and genetic content mimics that of parent cells, and is in part considered as surrogates of the parent tumor cells ${ }^{[18]}$. Moreover, TEXs can transfer messages from the parent tumor to recipient cells, including immune cells, within the $\mathrm{TME}^{[19]}$. 


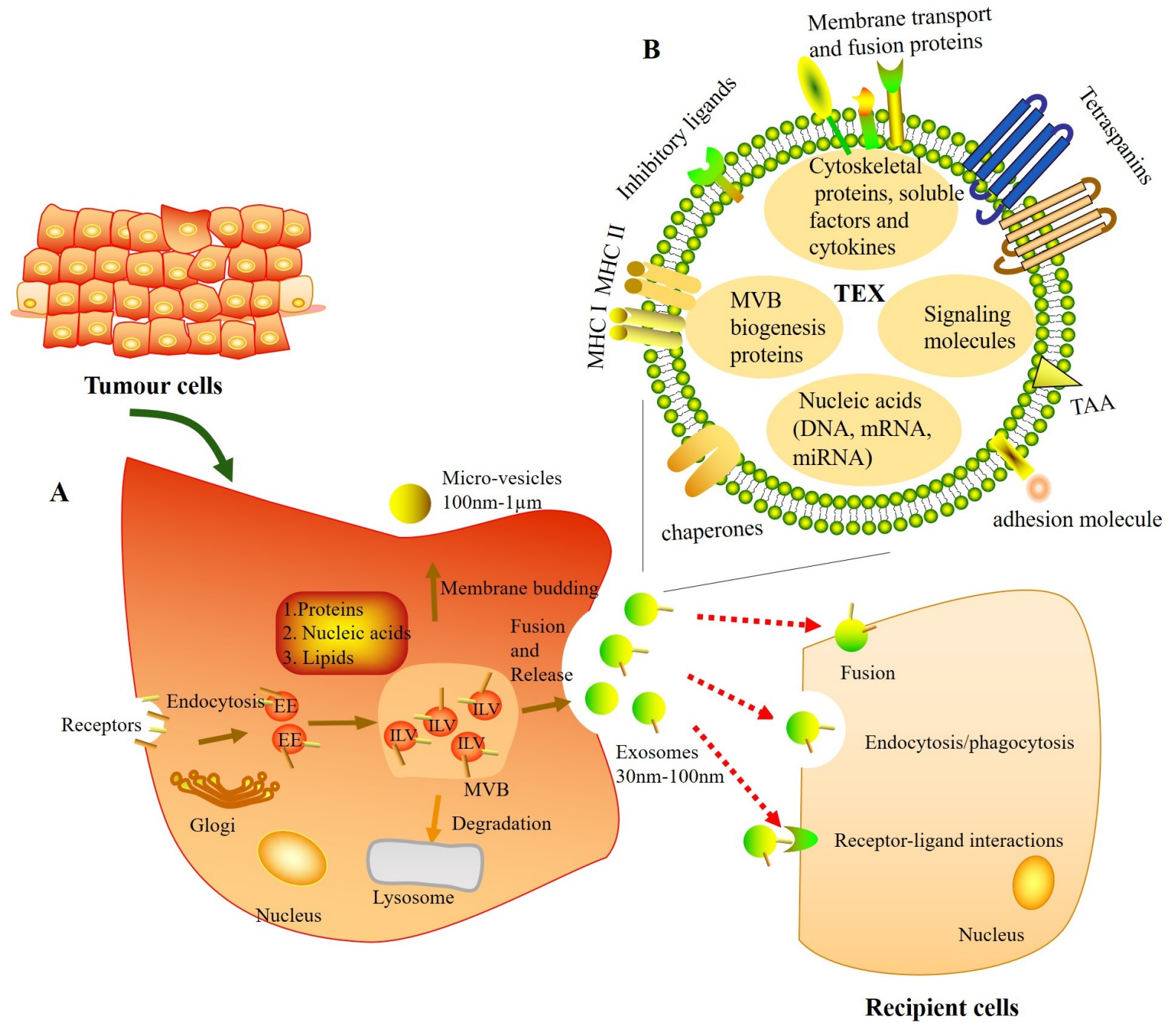

Figure 1. Molecular composition, biogenesis, release, and uptake of tumor-derived exosomes (TEXs). (A) TEXs originate from intraluminal vesicles (ILVs) in the multivesicular bodies (MVBs) (also known as late endosomes). Firstly, early endosomes (EEs) are formed when the membrane microdomains are endocytosed via inward budding of the plasma membrane. Then EEs mature into MVBs, which follow either fusion with the plasma membrane to form exosomes or degradation by lysosome. During this process, the proteins, nucleic acids, and lipids are packed into exosomes. Finally, exosomes can interact with recipient cells through three main ways: endocytosis/phagocytosis, direct fusion with cellular membrane, and receptor-ligand interactions. (B) Schematic diagram of components of TEXs.

\section{EFFECT OF TEXS FOR IMMUNE REGULATION IN LUNG CANCER}

Recently, TEXs have been proposed to act as crucial mediators between cellular communication by transferring both immunosuppressive and immunostimulatory signals to immune cells in a lung cancer microenvironment ${ }^{[20,21]}$ [Table 1, Figure 2].

\section{Immunosuppressive effect of TEXs}

TEXs modulate the activity of $\mathrm{T}$ cells by promoting apoptosis and inhibiting proliferation of $\mathrm{CD} 8^{+} \mathrm{T}$ cells ${ }^{[22]}$. Recent studies have indicated that TEXs contain PD-L1, which inhibits T cells activity and promotes tumor progression. Exosomes from lung cancer, melanoma, and breast cancer carry PD-L1 on their surface, which interacts with PD-1 via the extracellular domain, and thereby inactivate $\mathrm{T}_{\text {cells }}{ }^{[23]}$. Poggio et al. ${ }^{[24]}$ discovered that the majority of PD-L1 could be presented on the surface of TEXs, and genetic blockade of exosomal 
Table 1. Overview of exosomal cargo, source of exosomes, and their biological effects

\begin{tabular}{llll}
\hline Exosomal cargo & Donor & Biological effect & Ref. \\
\hline PD-L1 & H1299, H358, and H1264 & Inactivate T cells & {$[23]$} \\
EGFR & NSCLC biopsies & Induce tolerogenic DCs & {$[28]$} \\
miR-214 & Lewis lung carcinoma cells & Downregulate the PTEN-mediated signaling & {$[29]$} \\
miR-433 & Plasma of NSCLC patients & Inactivate the WNT/B catenin signaling & {$[31]$} \\
miR-21/29a & A-549 and SK-MES & Activate TLR7 and TLR8 & {$[32]$} \\
miR-21a & Lewis lung carcinoma cells & Promote MDSCs expansion & {$[51]$} \\
miR-103a & CL1-5 lung cancer cells & Activate of PI3K/Akt and STAT3 signaling pathways & {$[52]$} \\
circFARSA & A549 and PC9 cells & Polarize macrophages to the M2 phenotype & {$[54]$} \\
miR-770 & A549 cells & Suppress M2 macrophage polarization & {$[62]$} \\
\hline
\end{tabular}

NSCLC: Non small cell lung cancer; DCs: dendritic cells; MDSCs: myeloid-derived suppressor cells.

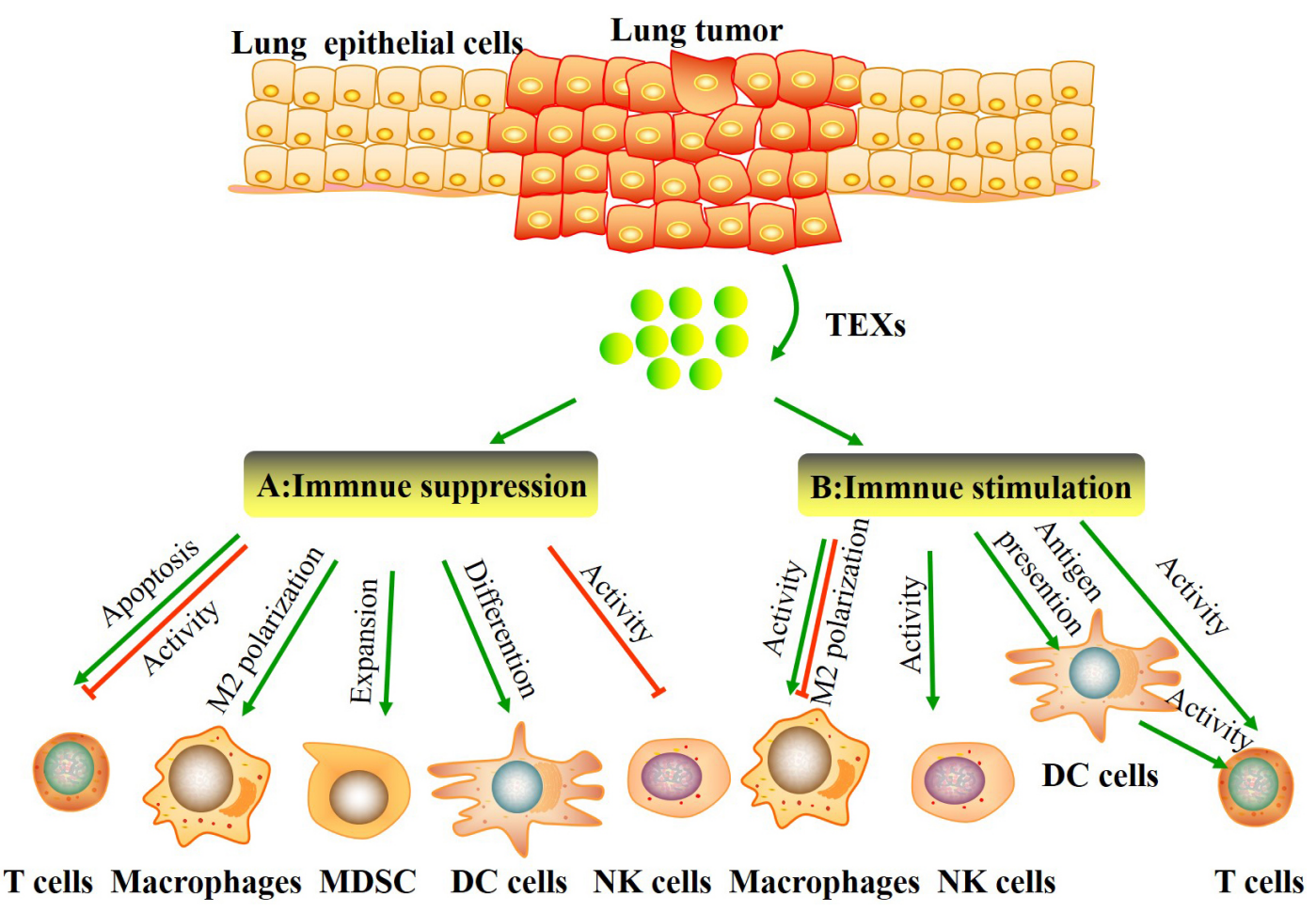

Figure 2. Tumor-derived exosomes (TEXs) carry and deliver both immunosuppressive and immunostimulatory signals to immune cells in the lung tumor microenvironment. (A) Immune suppression. TEXs contribute to establish an immunosuppressive TME by inducing apoptosis and inhibiting the activity of effector T cells, skewing M2 polarization of macrophages, expanding myeloid-derived suppressor cells (MDSCs), suppressing DCs differentiation, and impairing the function of NK cells. (B) Immune stimulation. TEXs can also stimulate immune cells to support antitumor activities, including enhancing the activity of macrophages and NK cells, suppressing M2 macrophage polarization, and increase T cells activity directly or indirectly.

PD-L1 could activate an anti-tumor immune response leading to extend survival in a subset of cancer patients. Moreover, it is suggested that TEXs with FasL expression could induce CD8 ${ }^{+} \mathrm{T}$ cell apoptosis ${ }^{[25,26]}$. Czystowska et al. ${ }^{[27]}$ uncovered the mechanism that the PI3K/Akt pathway was a central target for TEXs in regulating $\mathrm{CD}^{+} \mathrm{T}$ cell apoptosis. Huang et al. ${ }^{[28]}$ indicated that about $80 \%$ of exosomes isolated from nonsmall cell lung cancer (NSCLC) biopsies contained EGFR. These exosomes can be captured by dendritic cells (DCs). Then tolerogenic DCs were generated and induced tumor antigen specific regulatory T cells (Tregs), which could inhibit the function of tumor specific $\mathrm{CD} 8^{+} \mathrm{T}$ cells. Yin et al. ${ }^{[29]}$ found that miR-214 
was delivered into recipient $\mathrm{CD} 4^{+} \mathrm{T}$ cells via TEXs, so as to downregulate the PTEN-mediated signaling, thereby promoting Treg expansion and tumor growth. Interestingly, co-incubation of Treg with TEXs may enhance Treg number as well as its suppressive function with the increased production of inhibitory cytokines, TGF- $\beta 1$ and interleukin (IL)- $10^{[30]}$. Additionally, Liu et al. ${ }^{[31]}$ showed that exosome-derived miR433 inactivated the $\mathrm{WNT} / \beta$-catenin signaling pathway via targeting transmembrane p24 trafficking protein 5 , thus increasing infiltration of $\mathrm{CD} 4^{+}$and $\mathrm{CD} 8^{+}$cells in NSCLC.

Exosome-derived miR-21/29a derived from A-549 and SK-MES cells promoted lung cancer growth and metastasis through activating Toll like receptors TLR7 and TLR8 on immune cells including NK cells ${ }^{[32]}$. NK cells express a variety of receptors that are either stimulatory or inhibitory ${ }^{[33]}$. The downregulation of those stimulatory receptors, particularly NKG2D, may play an important role in decreasing activity of NK cells in lung cancer patients ${ }^{[34]}$. TEXs originating from hypoxic tumor cells deliver TGF- $\beta 1$ to NK cells, and thereby reduce $\mathrm{NKG}_{2} \mathrm{D}$ expression resulting in lower activity of NK cells ${ }^{[35]}$. TEXs can also attenuate NK cell activity via multiple mechanisms including shedding the NKG2D ligand on tumor cells, suppressing Janus kinase (Jak) 3 activation, inhibiting perforin or cyclin D3 production and down-regulation of IL-2-mediated pathways ${ }^{[36-41]}$. Moreover, TEX-carried MICA and MICB ligands can downregulate the stimulatory receptors, especially NKG2D on NK cells ${ }^{[42]}$.

TEXs suppressed the functioning of the immune system by affecting the monocyte differentiation and maturation ${ }^{[43,44]}$. TEXs were capable of blocking DCs migration to lymph nodes through inhibiting most C-C/C-X-C chemokine receptor expression ${ }^{[45]}$. Co-incubation of DCs with TEXs leaded to the down regulation of CD80 and CD86, and subsequently inhibited DCs maturation ${ }^{[46]}$. In addition, TEXs have been shown to induce the differentiation of myeloid precursor cells into highly suppressive myeloid-derived suppressor cells (MDSCs) ${ }^{[3,47]}$. Heat-shock protein 72 (Hsp72) on the TEXs surface could trigger the STAT3 activation and autocrine IL-6 production in MDSCs via a TLR2/MyD88-dependent manner, leading to the increment of immunosuppressive activity of $\mathrm{MDSCs}^{[48-50]}$. Zhang et al. ${ }^{[51]}$ indicated that miR-21a enriched in lung carcinoma cell-derived exosomes could promote MDSCs expansion via targeting PDCD4, thus enhancing tumor growth. Hsu et al. ${ }^{[52]}$ reported that miR-103a was upregulated in lung cancer derived exosomes under hypoxic conditions. Exosomal miR-103a could cause the activation of PI3K/Akt and STAT3 signaling pathways by directly targeting PTEN, thereby resulting in the enhancement of M2 polarization ${ }^{[52]}$. Additionally, the study with A549 (wild-type p53 allele) and H358 (p53 null allele) exosomes suggested that lung cancer cell-derived exosomes mediated M2 polarization may be p53 independent ${ }^{[53]}$. Another study showed that exosomal circFARSA was significantly upregulated in NSCLC tissues and stimulated NSCLC cell metastasis by polarizing macrophages to the M2 phenotype ${ }^{[54]}$.

Taken together, these data suggested that TEXs can modulate the immune response by transferring immunosuppressive signals to immune cells, which in turn contribute to tumor progression ${ }^{[7,34,55,56]}$.

\section{Immunostimulatory effect of TEXs}

TEXs have been reported to be involved in the suppression of the immune system in previous studies. However, since TEXs also carry stimulatory molecules that contribute to activating immune responses, recent studies also focused on anti-tumor immunity of exosomes.

TEXs can act as presenters participating in direct and indirect antigen presentation ${ }^{[57]}$. As direct presenters, TEXs present antigen to T cells via an MHC-peptide complex on their surface. On the other hand, TEXs can also indirectly transfer tumor antigen to antigen presenting cells, like DCs, and then activate the cytotoxic activity of $\mathrm{CD}^{+} \mathrm{T}$ cells and $\mathrm{CD} 4^{+} \mathrm{T}$ helper cells, so as to inhibit tumor growth ${ }^{[58]}$. Additionally, the 
enrichment of HSPs on TEXs such as Hsp70, can stimulate the activity of NK cells ${ }^{[59]}$ and macrophages ${ }^{[60]}$, and induce MHC class I-restricted cytotoxic T cells activation ${ }^{[61]}$. Tumor cell-derived exosomal miR-770 could suppress M2 macrophage polarization via targeting MAP3K1, which in turn decreased NSCLC tumor growth $^{[62]}$. Tetraspanins on the exosome surface mainly mediate cell adhesion and participate in maintaining the optimal conformation of immune proteins like MHC class II, thus playing an important role in antitumor immunity through exosomal targeting to DCs ${ }^{[61,63-65]}$.

Therefore, the effect of exosomes' immune stimulation depends mainly on their antigen presentation, while the effect of inhibiting immunity mainly depends on exosome-carried biological content consisting of ligands, miRNAs, and proteins, which may inhibit the cytotoxic activity of the NK and $\mathrm{CD}^{+} \mathrm{T}$ cells or increase suppressive immune cells such as MDSCs, Treg cells, and M2 macrophages. Understanding the effect of TEXs in immune regulation will allow for better understanding of the clinical application of TEXs in cancer diagnosis and treatment.

\section{TEXS SERVE AS DIAGNOSTIC AND PROGNOSTIC BIOMARKERS IN LUNG CANCER}

TEXs and their content in biofluids, which represent the content of parent cells, may serve as newly developed non-invasive biomarkers for diagnosis, prognosis, and monitoring the efficacy of treatment in lung cancer ${ }^{[9-11]}$.

Jakobsen et al. ${ }^{[66]}$ examined the potential of exosomal proteins as diagnostic markers in advanced NSCLC. The EV (extracellular vesicle) array showed that the expression levels of CD9, CD63, and CD81 were significantly high in cancerous patients. Likewise, according to the EV array, NYESO-1, EGFR, and PLAP showed a strong correlation with a poor survival in NSCLC ${ }^{[1]}$. In addition, SRGN, TPM3, THBS1, and HUWE1 may serve as biomarkers to distinguish lung adenocarcinoma subjects from controls ${ }^{[67]}$. Combination of carcinoembryonic antigen, exosomal alpha-2-HS-glycoprotein and extracellular matrix protein 1 (ECM1) could improve the diagnostic accuracy of NSCLC ${ }^{[68]}$. Gao et al. ${ }^{[69]}$ indicated that plasma exosomal total protein, Tim-3 and Galectin-9 were significantly increased in NSCLC, and were positively associated with larger tumor size, advanced TNM stage, and distant metastases. The higher level of leucinerich a-2-glycoprotein (LRG1) was detected in urinary exosomes and may be a non-invasive diagnostic biomarker of NSCLC in urine ${ }^{[70]}$.

Several studies have shown that exosomal miRNAs may serve as potential biomarkers for the early diagnosis of lung cancer. Rabinowits et al. ${ }^{[71]}$ suggested that exosomal miRNAs in NSCLC patients very closely resemble those in NSCLC tissue, indicating that such a liquid biopsy may obviate the need to obtain tumor tissues. Tumor-derived exosomal miRNAs, adenocarcinoma-specific miR-181-5p, miR-361-5p, miR-30a-3p, and miR-30e-3p, and squamous cell carcinoma-specific miR-10b-5p, miR-320b, and miR-15b$5 \mathrm{p}$, were isolated from the plasma of early-stage NSCLC patients, which are able to discriminate between adenocarcinoma and squamous cell carcinoma, thereby serving as noninvasive biomarkers for early diagnosis of NSCLC ${ }^{[72]}$. Exosomal miR-1169 and miR-260 have also been identified as potential biomarkers that can distinguish between early-stage wild-type EGFR and mutant EGFR NSCLC ${ }^{[73]}$. Exosomal miR-20b-5p and miR-3187-5p were drastically reduced in early-stage NSCLC patients than those in healthy controls, showing both exosomal miRNAs were efficient diagnostic biomarkers for early-stage NSCLC ${ }^{[74]}$. Exosomal miR-126 has also been identified as a possible diagnostic biomarker for NSCLC progression ${ }^{[75]}$. In fact, exosomes exist extensively in body fluids other than blood, and increasing attention has been focused on diagnostic assays in pleural fluid. Tamiya et al. ${ }^{[76]}$ revealed that exosomal miR-182 and miR-210 were demonstrated to have a diagnostic potential for differentiating lung adenocarcinoma pleural effusion from benign pleural effusion. Additionally, exosomal miR-200 and mRNA transcript encoding lipocalin-2 from 
pleural effusions may be considered as diagnostic markers to discriminate lung adenocarcinoma patients from patients with benign inflammatory processes ${ }^{[77]}$.

Recently, exosomal RNAs have been reported to predict the prognosis of a variety of cancers including lung cancer. Dejima et al..$^{[78]}$ showed that exosomal miR-21 and miR-4257 levels of the NSCLC patients were significantly upregulated during recurrence and can be used as recurrence-specific biomarkers. Additionally, another study reported that low exosomal let-7a-5p levels were significantly associated with a worse cancer-related survival rate in lung adenocarcinoma patients ${ }^{[79]}$. Luo et al.$^{\left[{ }^{[0]}\right]}$ also demonstrated that serum exosomal miR-382 was considered as an independent prognostic biomarker for NSCLC. Zhang et al. ${ }^{\left[{ }^{[1]}\right.}$ suggested that exosomal lncRNA MALAT-1 was highly expressed in NSCLC patients and was positively associated with lymphatic metastasis and TNM stage, thereby indicating that exosomal MALAT-1 may be a non-invasive biomarker for diagnosis and prognosis of NSCLC. Moreover, tumorderived exosomal eIF4E has the potential for use as a biomarker for survival prediction in NSCLC ${ }^{[82]}$.

Acquired resistance to general therapies, including chemotherapy, radiotherapy, immunotherapy, and targeted therapy, is a major challenge in the treatment of lung cancer. Nowadays, the use of exosomes as biomarkers for predicting therapeutic responses has gathered much attention. Exosomal hsa_circ_0014235 isolated from plasma promoted cisplatin chemoresistance and may serve as a promising biomarker for NSCLC treatment ${ }^{[83]}$. Exosomal miR-4443 might also promote cisplatin resistance of NSCLC by regulating FSP1-mediated ferroptosis ${ }^{[84]}$. In addition, Li et al. ${ }^{[85]}$ showed that plasma exosomal miR-92b-3p was significantly increased in chemoresistant small cell lung cancer patients and might serve as a potential dynamic biomarker for monitoring the drug resistance. Exosomal miR-29a-3p and miR-150-5p were identified as circulating biomarkers during thoracic radiation therapy for NSCLC and were correlated with delivered radiation therapy dose ${ }^{[86]}$. At present, precision medicine based on immunotherapy and targeted therapy has given new hope for lung cancer patients. The ensuing problems of drug resistance have gained much interest from the research community. For example, exosomal miR-323-3p, miR-1468-3p, miR-5189-5p, and miR-6513-5p were identified as potential biomarkers to discriminate between osimertinib-resistant and osimertinib-sensitive NSCLC patients ${ }^{[87]}$, and exosomal miR-210-3p secreted by osimertinib-resistant HCC827 and PC-9 cells was able to promote epithelial-mesenchymal transition and drug resistance in osimertinib-sensitive, EGFR mutant NSCLC cells ${ }^{[88]}$. Moreover, exosomal circRNA_102481 enhanced EGFR-TKIs resistance through the microRNA-30a-5p/ROR1 axis in NSCLC ${ }^{[89]}$. Peng et al. ${ }^{\left[{ }^{[0]}\right.}$ suggested that high exosomal miR320d, miR320c, and miR320b levels were corelated with poor response to anti-PD-1 treatment in patients with NSCLC, and exosomal miR-125b-5p was identified to be one potential target for anti-PD-1 treatment. In summary, exosomal RNAs in lung cancer could be used for monitoring therapy response/relapse to improve personalized therapy strategies.

\section{THE ROLE OF TEXS IN IMMUNOTHERAPY OF LUNG CANCER}

\section{Effect of TEXs for resistance to immunotherapy}

TEXs may suppress proliferation and differentiation of immune cells and have the ability to influence their biological function. TEXs carrying a variety of tumor associated antigens or immunoinhibitory mediators not only suppress antitumor functions of immune effector cells, but also appear to impede effective response to immunotherapy in cancer $^{[8]}$. Tumor associated antigens on TEXs could efficiently bind antibodies produced against cancer cells and block the access of therapeutic antibodies to the cancer cells, leading to a decrease in effectiveness of cancer therapy ${ }^{[91]}$. Additionally, TEXs are able to inhibit antibody dependent cell-mediated cytotoxicity, which serves as a critical mechanism of therapeutic antitumor activity of anticancer antibodies ${ }^{[91]}$. During therapy, immune escape in NSCLC occurs through a multistep process that facilitates tumor growth and progression. Acquired tumor resistance to immunotherapy could be 
directly reflected in the production of TEXs ${ }^{[92]}$. Most importantly, Kim et al. ${ }^{[93]}$ showed that lung cancer cells increased their production of immunosuppressive exosomes during acquired resistance to anti-PDL1 immunotherapy.

\section{TEXs-based cancer vaccine and immunotherapy}

TEXs are nanoscale membrane-derived vesicles that are thought to be important mediators of intercellular communication. Moreover, TEXs with distinct characteristics such as stability, permeability, biocompatibility, low immunogenicity, and low toxicity can efficiently deliver tumor antigens to DCs, thus they can be used as self-antigen carriers to stimulate immune respons $\mathrm{e}^{[94-96]}$. Increasing evidences have demonstrated that the activation and maturation of DCs by TEXs could enhance anti-tumor effects and may be applied for lung cancer immunotherapy. For example, TEXs from CD4oL-gene modified 3LL lung tumor cells have the potent ability to activate DCs, resulting in significantly increased tumor antigenspecific $\mathrm{CD} 4^{+} \mathrm{T}$ cell proliferation and $\mathrm{CD} 8^{+} \mathrm{T}$ cell responses, revealing a powerful antitumor effect ${ }^{[97]}$. In addition, the exosomes derived from Rab27-overexpressing NSCLC cells also stimulated the proliferation and maturation of DCs effectively, promoted $\mathrm{CD}^{+} \mathrm{T}$ cell proliferation and elicited potent antitumor immune responses ${ }^{[98]}$. Multiple studies have already proved that TEXs which were used as tumor antigens source for DC vaccines, have greater efficacy and safety than conventional tumor cell lysates ${ }^{[99-103]}$. Wang et al ${ }^{[104]}$ indicated that TEXs were more potent than tumor cell lysates to trigger DC-mediated immune responses and decrease Tregs, contributing to improving vaccine-elicited immunotherapy for lung cancer. Thus, DCs loaded with TEXs may be promising therapeutics without severe side effects and treatment resistance in clinical application ${ }^{[105]}$.

\section{CONCLUSION}

TEXs are important mediators of intercellular communication and have been proven to play a key role in the TME. The biogenesis and secretion of TEXs have been widely reported. They carry a variety of cargoes and are involved in both immunosuppressive and immunostimulatory signaling pathways by delivering molecular signals to immune cells. The small size of TEXs and their contents render them highly interesting for biomedical applications, such as biomarker molecules and anticancer vaccines. Based on the data herein, we suggest that TEXs could be manipulated to provide clinical benefits and improve the clinical management of lung cancer.

\section{DECLARATIONS}

\section{Authors' contributions}

Manuscript writing: Wu J

Manuscript revision: Li S

Study design: Zhang P

All authors read and approved the final manuscript.

\section{Availability of data and materials}

Not applicable.

\section{Financial support and sponsorship}

None.

\section{Conflicts of interest}

All authors declared that there are no conflicts of interest. 


\section{Ethical approval and consent to participate}

Not applicable.

\section{Consent for publication}

Not applicable.

\section{Copyright}

(C) The Author(s) 2022.

\section{REFERENCES}

1. Herbst RS, Morgensztern D, Boshoff C. The biology and management of non-small cell lung cancer. Nature 2018;553:446-54. DOI PubMed

2. Gadgeel S, Rodríguez-Abreu D, Speranza G, et al. Updated analysis from KEYNOTE-189: pembrolizumab or placebo plus pemetrexed and platinum for previously untreated metastatic nonsquamous non-small-cell lung cancer. J Clin Oncol 2020;38:150517. DOI PubMed

3. Mok TSK, Wu Y, Kudaba I, et al. Pembrolizumab versus chemotherapy for previously untreated, PD-L1-expressing, locally advanced or metastatic non-small-cell lung cancer (KEYNOTE-042): a randomised, open-label, controlled, phase 3 trial. Lancet 2019;393:1819-30. DOI PubMed

4. Cao C, Guo A, Chen C, Zielinski R, Bott M. Neoadjuvant immunotherapy for patients with non-small cell lung cancer-current evidence. Ann Transl Med 2020;8:1476. DOI PubMed PMC

5. Xu Z, Zeng S, Gong Z, Yan Y. Exosome-based immunotherapy: a promising approach for cancer treatment. Mol Cancer 2020;19:160. DOI PubMed PMC

6. Zhu L, Sun HT, Wang S, et al. Isolation and characterization of exosomes for cancer research. J Hematol Oncol 2020;13:152. DOI PubMed PMC

7. Olejarz W, Dominiak A, Żołnierzak A, Kubiak-Tomaszewska G, Lorenc T. Tumor-derived exosomes in immunosuppression and immunotherapy. J Immunol Res 2020;2020:6272498. DOI PubMed PMC

8. Whiteside TL. Tumor-derived exosomes and their role in cancer progression. Elsevier; 2016. p. 103-41.

9. Soung YH, Ford S, Zhang V, Chung J. Exosomes in cancer diagnostics. Cancers (Basel) 2017;9:8. DOI PubMed PMC

10. Sharma S, Salomon C. Techniques associated with exosome isolation for biomarker development: liquid biopsies for ovarian cancer detection. In: Thurin M, Cesano A, Marincola FM, editors. Biomarkers for immunotherapy of cancer. New York: Springer; 2020. p. 181-99.

11. Sandfeld-Paulsen B, Aggerholm-Pedersen N, Bæk R, et al. Exosomal proteins as prognostic biomarkers in non-small cell lung cancer. Mol Oncol 2016;10:1595-602. DOI PubMed PMC

12. Tian X, Shen H, Li Z, Wang T, Wang S. Tumor-derived exosomes, myeloid-derived suppressor cells, and tumor microenvironment. $J$ Hematol Oncol 2019;12:84. DOI PubMed PMC

13. Xie F, Zhou X, Fang M, et al. Extracellular vesicles in cancer immune microenvironment and cancer immunotherapy. Adv Sci (Weinh) 2019;6:1901779. DOI PubMed PMC

14. Salimi L, Akbari A, Jabbari N, et al. Synergies in exosomes and autophagy pathways for cellular homeostasis and metastasis of tumor cells. Cell Biosci 2020;10:64. DOI PubMed PMC

15. Taghikhani A, Farzaneh F, Sharifzad F, Mardpour S, Ebrahimi M, Hassan ZM. Engineered tumor-derived extracellular vesicles: potentials in cancer immunotherapy. Front Immunol 2020;11:221. DOI PubMed PMC

16. Gupta A, Pulliam L. Exosomes as mediators of neuroinflammation. J Neuroinflammation 2014;11:68. DOI PubMed PMC

17. Czystowska-Kuzmicz M, Whiteside TL. The potential role of tumor-derived exosomes in diagnosis, prognosis, and response to therapy in cancer. Expert Opin Biol Ther 2021;21:241-58. DOI PubMed PMC

18. Whiteside TL. The effect of tumor-derived exosomes on immune regulation and cancer immunotherapy. Future Oncol 2017;13:258392. DOI PubMed PMC

19. Whiteside TL. The emerging role of plasma exosomes in diagnosis, prognosis and therapies of patients with cancer. Contemp Oncol (Pozn) 2018;22:38-40. DOI PubMed PMC

20. Chen R, Xu X, Qian Z, et al. The biological functions and clinical applications of exosomes in lung cancer. Cell Mol Life Sci 2019;76:4613-33. DOI PubMed

21. Hu C, Meiners S, Lukas C, Stathopoulos GT, Chen J. Role of exosomal microRNAs in lung cancer biology and clinical applications. Cell Prolif 2020;53:e12828. DOI PubMed PMC

22. Wieckowski EU, Visus C, Szajnik M, Szczepanski MJ, Storkus WJ, Whiteside TL. Tumor-derived microvesicles promote regulatory $\mathrm{T}$ cell expansion and induce apoptosis in tumor-reactive activated CD8+ T lymphocytes. J Immunol 2009;183:3720-30. DOI PubMed PMC

23. Chen G, Huang AC, Zhang W, et al. Exosomal PD-L1 contributes to immunosuppression and is associated with anti-PD-1 response. Nature 2018;560:382-6. DOI PubMed PMC

24. Poggio M, Hu T, Pai CC, et al. Suppression of exosomal PD-L1 induces systemic anti-tumor immunity and memory. Cell 2019;177:414-27.e13. DOI PubMed PMC 
25. Kim JW, Wieckowski E, Taylor DD, Reichert TE, Watkins S, Whiteside TL. Fas ligand-positive membranous vesicles isolated from sera of patients with oral cancer induce apoptosis of activated T lymphocytes. Clin Cancer Res 2005;11:1010-20. PubMed

26. Abusamra AJ, Zhong Z, Zheng X, et al. Tumor exosomes expressing Fas ligand mediate CD8+ T-cell apoptosis. Blood Cells Mol Dis 2005;35:169-73. DOI PubMed

27. Czystowska M, Han J, Szczepanski MJ, et al. IRX-2, a novel immunotherapeutic, protects human T cells from tumor-induced cell death. Cell Death Differ 2009;16:708-18. DOI PubMed PMC

28. Huang SH, Li Y, Zhang J, Rong J, Ye S. Epidermal growth factor receptor-containing exosomes induce tumor-specific regulatory T cells. Cancer Invest 2013;31:330-5. DOI PubMed

29. Yin Y, Cai X, Chen X, et al. Tumor-secreted miR-214 induces regulatory T cells: a major link between immune evasion and tumor growth. Cell Res 2014;24:1164-80. DOI PubMed PMC

30. Szajnik M, Czystowska M, Szczepanski MJ, Mandapathil M, Whiteside TL. Tumor-derived microvesicles induce, expand and upregulate biological activities of human regulatory T cells (Treg). PLoS One 2010;5:e11469. DOI PubMed PMC

31. Liu B, Zhang R, Zhu Y, Hao R. Exosome-derived microRNA-433 inhibits tumorigenesis through incremental infiltration of CD4 and CD8 cells in non-small cell lung cancer. Oncol Lett 2021;22:607. DOI PubMed PMC

32. Fabbri M, Paone A, Calore F, et al. MicroRNAs bind to toll-like receptors to induce prometastatic inflammatory response. Proc Natl Acad Sci U S A 2012;109:E2110-6. DOI PubMed PMC

33. Marcus A, Gowen BG, Thompson TW, et al. Recognition of tumors by the innate immune system and natural killer cells. $A d v$ Immunol 2014;122:91-128. DOI PubMed PMC

34. Alipoor SD, Mortaz E, Varahram M, et al. The potential biomarkers and immunological effects of tumor-derived exosomes in lung cancer. Front Immunol 2018;9:819. DOI PubMed PMC

35. Berchem G, Noman MZ, Bosseler M, et al. Hypoxic tumor-derived microvesicles negatively regulate NK cell function by a mechanism involving TGF- $\beta$ and miR23a transfer. Oncoimmunology 2016;5:e1062968. DOI PubMed PMC

36. Whiteside TL. Immune modulation of T-cell and NK (natural killer) cell activities by TEXs (tumour-derived exosomes). Biochem Soc Trans 2013;41:245-51. DOI PubMed PMC

37. Clayton A, Mitchell JP, Court J, Linnane S, Mason MD, Tabi Z. Human tumor-derived exosomes down-modulate NKG2D expression. J Immunol 2008;180:7249-58. DOI PubMed

38. Ashiru O, Boutet P, Fernández-Messina L, et al. Natural killer cell cytotoxicity is suppressed by exposure to the human NKG2D ligand MICA*008 that is shed by tumor cells in exosomes. Cancer Res 2010;70:481-9. DOI PubMed PMC

39. Liu Y, Xiang X, Zhuang X, et al. Contribution of MyD88 to the tumor exosome-mediated induction of myeloid derived suppressor cells. Am J Pathol 2010;176:2490-9. DOI PubMed PMC

40. Deng W, Gowen BG, Zhang L, et al. Antitumor immunity. A shed NKG2D ligand that promotes natural killer cell activation and tumor rejection. Science 2015;348:136-9. DOI PubMed PMC

41. Filipazzi P, Bürdek M, Villa A, Rivoltini L, Huber V. Recent advances on the role of tumor exosomes in immunosuppression and disease progression. Semin Cancer Biol 2012;22:342-9. DOI PubMed

42. Szczepanski MJ, Szajnik M, Welsh A, Whiteside TL, Boyiadzis M. Blast-derived microvesicles in sera from patients with acute myeloid leukemia suppress natural killer cell function via membrane-associated transforming growth factor-beta1. Haematologica 2011;96:1302-9. DOI PubMed PMC

43. Kunigelis KE, Graner MW. The dichotomy of tumor exosomes (TEX) in cancer immunity: is it all in the ConTEXt? Vaccines (Basel) 2015;3:1019-51. DOI PubMed PMC

44. Hosseini R, Asef-Kabiri L, Yousefi H, et al. The roles of tumor-derived exosomes in altered differentiation, maturation and function of dendritic cells. Mol Cancer 2021;20:83. DOI PubMed PMC

45. Ning Y, Shen K, Wu Q, et al. Tumor exosomes block dendritic cells maturation to decrease the T cell immune response. Immunol Lett 2018;199:36-43. DOI PubMed

46. Ludwig S, Sharma P, Theodoraki MN, et al. Molecular and functional profiles of exosomes from HPV(+) and HPV(-) head and neck cancer cell lines. Front Oncol 2018;8:445. DOI PubMed PMC

47. Xiang X, Poliakov A, Liu C, et al. Induction of myeloid-derived suppressor cells by tumor exosomes. Int J Cancer 2009;124:262133. DOI PubMed PMC

48. Chalmin F, Ladoire S, Mignot G, et al. Membrane-associated Hsp72 from tumor-derived exosomes mediates STAT3-dependent immunosuppressive function of mouse and human myeloid-derived suppressor cells. J Clin Invest 2010;120:457-71. DOI PubMed PMC

49. Mignot G, Chalmin F, Ladoire S, Rébé C, Ghiringhelli F. Tumor exosome-mediated MDSC activation. Am J Pathol 2011;178:14034; author reply 1404-5. DOI PubMed PMC

50. Xiang X, Liu Y, Zhuang X, et al. TLR2-mediated expansion of MDSCs is dependent on the source of tumor exosomes. Am J Pathol 2010;177:1606-10. DOI PubMed PMC

51. Zhang X, Li F, Tang Y, et al. miR-21a in exosomes from Lewis lung carcinoma cells accelerates tumor growth through targeting PDCD4 to enhance expansion of myeloid-derived suppressor cells. Oncogene 2020;39:6354-69. DOI PubMed

52. Hsu YL, Hung JY, Chang WA, et al. Hypoxic lung-cancer-derived extracellular vesicle microRNA-103a increases the oncogenic effects of macrophages by targeting PTEN. Mol Ther 2018;26:568-81. DOI PubMed PMC

53. Pritchard A, Tousif S, Wang Y, et al. Lung tumor cell-derived exosomes promote M2 macrophage polarization. Cells 2020;9:1303. DOI PubMed PMC

54. Chen T, Liu Y, Li C, et al. Tumor-derived exosomal circFARSA mediates M2 macrophage polarization via the PTEN/PI3K/AKT 
pathway to promote non-small cell lung cancer metastasis. Cancer Treat Res Commun 2021;28:100412. DOI PubMed

55. Zhang L, Yu D. Exosomes in cancer development, metastasis, and immunity. Biochim Biophys Acta Rev Cancer 2019;1871:455-68. DOI PubMed PMC

56. Whiteside TL, Diergaarde B, Hong CS. Tumor-derived exosomes (TEX) and their role in immuno-oncology. Int $J$ Mol Sci 2021;22:6234. DOI PubMed PMC

57. Chaput N, Théry C. Exosomes: immune properties and potential clinical implementations. Semin Immunopathol 2011;33:419-40. DOI PubMed

58. Greening DW, Gopal SK, Xu R, Simpson RJ, Chen W. Exosomes and their roles in immune regulation and cancer. Semin Cell Dev Biol 2015;40:72-81. DOI PubMed

59. Gastpar R, Gehrmann M, Bausero MA, et al. Heat shock protein 70 surface-positive tumor exosomes stimulate migratory and cytolytic activity of natural killer cells. Cancer Res 2005;65:5238-47. DOI PubMed PMC

60. Vega VL, Rodríguez-Silva M, Frey T, et al. Hsp70 translocates into the plasma membrane after stress and is released into the extracellular environment in a membrane-associated form that activates macrophages. J Immunol 2008;180:4299-307. DOI PubMed

61. Li XB, Zhang ZR, Schluesener HJ, Xu SQ. Role of exosomes in immune regulation. J Cell Mol Med 2006;10:364-75. DOI PubMed PMC

62. Liu J, Luo R, Wang J, et al. Tumor cell-derived exosomal miR-770 inhibits M2 macrophage polarization via targeting MAP3K1 to Inhibit the invasion of non-small cell lung cancer cells. Front Cell Dev Biol 2021;9:679658. DOI PubMed PMC

63. Jiang L, Gu Y, Du Y, Liu J. Exosomes: diagnostic biomarkers and therapeutic delivery vehicles for cancer. Mol Pharm 2019;16:3333-49. DOI PubMed

64. Nedaeinia R, Manian M, Jazayeri MH, et al. Circulating exosomes and exosomal microRNAs as biomarkers in gastrointestinal cancer. Cancer Gene Ther 2017;24:48-56. DOI PubMed

65. Shan Z, Wang H, Zhang Y, Min W. The role of tumor-derived exosomes in the abscopal effect and immunotherapy. Life (Basel) 2021;11:381. DOI PubMed PMC

66. Jakobsen KR, Paulsen BS, Bæk R, Varming K, Sorensen BS, Jørgensen MM. Exosomal proteins as potential diagnostic markers in advanced non-small cell lung carcinoma. J Extracell Vesicles 2015;4:26659. DOI PubMed PMC

67. Vykoukal J, Sun N, Aguilar-Bonavides C, et al. Plasma-derived extracellular vesicle proteins as a source of biomarkers for lung adenocarcinoma. Oncotarget 2017;8:95466-80. DOI PubMed PMC

68. Niu L, Song X, Wang N, Xue L, Song X, Xie L. Tumor-derived exosomal proteins as diagnostic biomarkers in non-small cell lung cancer. Cancer Sci 2019;110:433-42. DOI PubMed PMC

69. Gao J, Qiu X, Li X, et al. Expression profiles and clinical value of plasma exosomal Tim-3 and Galectin-9 in non-small cell lung cancer. Biochem Biophys Res Commun 2018;498:409-15. DOI PubMed

70. Li Y, Zhang Y, Qiu F, Qiu Z. Proteomic identification of exosomal LRG1: a potential urinary biomarker for detecting NSCLC. Electrophoresis 2011;32:1976-83. DOI PubMed

71. Rabinowits G, Gerçel-Taylor C, Day JM, Taylor DD, Kloecker GH. Exosomal microRNA: a diagnostic marker for lung cancer. Clin Lung Cancer 2009;10:42-6. DOI PubMed

72. Jin X, Chen Y, Chen H, et al. Evaluation of tumor-derived exosomal miRNA as potential diagnostic biomarkers for early-stage nonsmall cell lung cancer using next-generation sequencing. Clin Cancer Res 2017;23:5311-9. DOI PubMed

73. Xia J, Luo M, Dai L, Wang L, Wang L, Zhu J. Serum exosomal microRNAs as predictive markers for EGFR mutations in non-smallcell lung cancer. J Clin Lab Anal 2021;35:e23743. DOI PubMed PMC

74. Zhang ZJ, Song XG, Xie L, et al. Circulating serum exosomal miR-20b-5p and miR-3187-5p as efficient diagnostic biomarkers for early-stage non-small cell lung cancer. Exp Biol Med (Maywood) 2020;245:1428-36. DOI PubMed PMC

75. Grimolizzi F, Monaco F, Leoni F, et al. Exosomal miR-126 as a circulating biomarker in non-small-cell lung cancer regulating cancer progression. Sci Rep 2017;7:15277. DOI PubMed PMC

76. Tamiya H, Mitani A, Saito A, et al. Exosomal MicroRNA expression profiling in patients with lung adenocarcinoma-associated malignant pleural effusion. Anticancer Res 2018;38:6707-14. DOI PubMed

77. Hydbring P, De Petris L, Zhang Y, et al. Exosomal RNA-profiling of pleural effusions identifies adenocarcinoma patients through elevated miR-200 and LCN2 expression. Lung Cancer 2018;124:45-52. DOI PubMed

78. Dejima H, Iinuma H, Kanaoka R, Matsutani N, Kawamura M. Exosomal microRNA in plasma as a non-invasive biomarker for the recurrence of non-small cell lung cancer. Oncol Lett 2017;13:1256-63. DOI PubMed PMC

79. Zhang L, Hao C, Zhai R, et al. Downregulation of exosomal let-7a-5p in dust exposed- workers contributes to lung cancer development. Respir Res 2018;19:235. DOI PubMed PMC

80. Luo R, Liu H, Chen J. Reduced circulating exosomal miR-382 predicts unfavorable outcome in non-small cell lung cancer. Int J Clin Exp Pathol 2021;14:469-74. PubMed PMC

81. Zhang R, Xia Y, Wang Z, et al. Serum long non coding RNA MALAT-1 protected by exosomes is up-regulated and promotes cell proliferation and migration in non-small cell lung cancer. Biochem Biophys Res Commun 2017;490:406-14. DOI PubMed

82. Dong Q, Dong L, Liu S, Kong Y, Zhang M, Wang X. Tumor-derived exosomal eIF4E as a biomarker for survival prediction in patients with non-small cell lung cancer. Med Sci Monit 2020;26:e923210. DOI PubMed PMC

83. Xu X, Tao R, Sun L, Ji X. Exosome-transferred hsa_circ_0014235 promotes DDP chemoresistance and deteriorates the development of non-small cell lung cancer by mediating the miR-520a-5p/CDK4 pathway. Cancer Cell Int 2020;20:552. DOI PubMed PMC

84. Song Z, Jia G, Ma P, Cang S. Exosomal miR-4443 promotes cisplatin resistance in non-small cell lung carcinoma by regulating FSP1 m6A modification-mediated ferroptosis. Life Sci 2021;276:119399. DOI PubMed 
85. Li M, Shan W, Hua Y, et al. Exosomal miR-92b-3p promotes chemoresistance of small cell lung cancer through the PTEN/AKT pathway. Front Cell Dev Biol 2021;9:661602. DOI PubMed PMC

86. Dinh TK, Fendler W, Chałubińska-Fendler J, et al. Circulating miR-29a and miR-150 correlate with delivered dose during thoracic radiation therapy for non-small cell lung cancer. Radiat Oncol 2016;11:61. DOI PubMed PMC

87. Janpipatkul K, Trachu N, Watcharenwong P, et al. Exosomal microRNAs as potential biomarkers for osimertinib resistance of nonsmall cell lung cancer patients. Cancer Biomark 2021;31:281-94. DOI PubMed

88. Hisakane K, Seike M, Sugano T, et al. Exosome-derived miR-210 involved in resistance to osimertinib and epithelial-mesenchymal transition in EGFR mutant non-small cell lung cancer cells. Thorac Cancer 2021;12:1690-8. DOI PubMed PMC

89. Yang B, Teng F, Chang L, et al. Tumor-derived exosomal circRNA_102481 contributes to EGFR-TKIs resistance via the miR-30a5p/ROR1 axis in non-small cell lung cancer. Aging (Albany NY) 2021;13:13264-86. DOI PubMed PMC

90. Peng XX, Yu R, Wu X, et al. Correlation of plasma exosomal microRNAs with the efficacy of immunotherapy in EGFR/ALK wildtype advanced non-small cell lung cancer. J Immunother Cancer 2020;8:e000376. DOI

91. Battke C, Ruiss R, Welsch U, et al. Tumour exosomes inhibit binding of tumour-reactive antibodies to tumour cells and reduce ADCC. Cancer Immunol Immunother 2011;60:639-48. DOI PubMed

92. Meehan K, Vella LJ. The contribution of tumour-derived exosomes to the hallmarks of cancer. Crit Rev Clin Lab Sci 2016;53:121-31. DOI PubMed

93. Kim DH, Kim H, Choi YJ, et al. Exosomal PD-L1 promotes tumor growth through immune escape in non-small cell lung cancer. Exp Mol Med 2019;51:1-13. DOI PubMed PMC

94. Pitt JM, Charrier M, Viaud S, et al. Dendritic cell-derived exosomes as immunotherapies in the fight against cancer. J Immunol 2014;193:1006-11. DOI PubMed

95. Batrakova EV, Kim MS. Using exosomes, naturally-equipped nanocarriers, for drug delivery. J Control Release 2015;219:396-405. DOI PubMed PMC

96. van der Pol E, Böing AN, Harrison P, Sturk A, Nieuwland R. Classification, functions, and clinical relevance of extracellular vesicles. Pharmacol Rev 2012;64:676-705. DOI PubMed

97. Wang J, Wang L, Lin Z, Tao L, Chen M. More efficient induction of antitumor T cell immunity by exosomes from CD40L genemodified lung tumor cells. Mol Med Rep 2014;9:125-31. DOI PubMed

98. Li W, Mu D, Tian F, et al. Exosomes derived from Rab27aoverexpressing tumor cells elicit efficient induction of antitumor immunity. Mol Med Rep 2013;8:1876-82. DOI PubMed

99. Raposo G, Nijman HW, Stoorvogel W, et al. B lymphocytes secrete antigen-presenting vesicles. J Exp Med 1996;183:1161-72. DOI PubMed PMC

100. Zitvogel L, Regnault A, Lozier A, et al. Eradication of established murine tumors using a novel cell-free vaccine: dendritic cellderived exosomes. Nat Med 1998;4:594-600. DOI PubMed

101. Lee EY, Park KS, Yoon YJ, et al. Therapeutic effects of autologous tumor-derived nanovesicles on melanoma growth and metastasis. PLoS One 2012;7:e33330. DOI PubMed PMC

102. Marton A, Vizler C, Kusz E, et al. Melanoma cell-derived exosomes alter macrophage and dendritic cell functions in vitro. Immunol Lett 2012;148:34-8. DOI PubMed

103. Gu X, Erb U, Büchler MW, Zöller M. Improved vaccine efficacy of tumor exosome compared to tumor lysate loaded dendritic cells in mice. Int J Cancer 2015;136:E74-84. DOI PubMed

104. Wang C, Huang X, Wu Y, Wang J, Li F, Guo G. Tumor cell-associated exosomes robustly elicit anti-tumor immune responses through modulating dendritic cell vaccines in lung tumor. Int J Biol Sci 2020;16:633-43. DOI PubMed PMC

105. Liu H, Chen L, Peng Y, et al. Dendritic cells loaded with tumor derived exosomes for cancer immunotherapy. Oncotarget 2018;9:2887-94. DOI PubMed PMC 